dicating muscularity, when physiologically considered. I will now make some observations upon the function of the "pecten," which I believe will not only show what thate unction really is, but which will also corroborate the correctness of the explanation above given of the use of the ciliary processes.

It may at first sight appear rather at variance with the explanation above proposed, respecting the mechanism of the ciliary processes, that these parts should be comparatively so little developed in the class of animals in which the ciliary muscle is most perfect, and in which also the adapting power of the eye is considered to be very great. It also seems some. what at variance with my explanation, that there should not be some correspondence between the development of the ciliary musele and the optical perfection of the lens, which, comparing these parts in the eyes of birds and mammals, does not appear to be the case. (The lens of the bird is much less dense, especially at its centre, than that of the mammal. This can be seen by drying the bird's lens, when it will be found to shrink more than that of the mammal, and, in the place of preserving its convexity, to become very much indented at its centre.) For if the adjusting power of the eye depended wholly upon the motion of the lens, one would expect to find co-existing with a lens of inferior density, (and consequently of longer focus, and with a larger ciliary muscle, also larger ciliary processes, the distance between the geometrical focus of parallel and divergent rays being required to vary more under the same circumstances than if the focal distance of the lens were less, (see Wood's or any other work on Optics.) But if such had been the case in the eye of the bird, then birds must necessarily have had a more globular form of eye than mammals, admitting that the form of the eye of the latter is just what it onght to be; whilst, on the contrary, the eye of the bird is required to be much more flat, in consequence of the form and size of the head; therefore some other means must be looked for in the bird, to aid those already described, for adapting the eye to distances. Now, it must be recollected that in the eyes of birds, lizards, and some other species of reptiles, there is, besides ciliary processes, a distinct organ called "pecten," that it has the same structure as the ciliary processes, and that where one is large the other is small, and the reverse; and therefore it seems not impossible that the pecten performs a function similar to that performed by the ciliary processes.

Before considering further the office of the pecten, it will be necessary to give a brief description of its structure and situation. The pecten is a plicated vascular membrane, present only in the eyes of birds, the larger kinds of lizards, and some other reptiles. In the common fowl it is situated at the inferior and outer part of the eye, extending from the entrance of the optic nerve, with which it is very much connected, almost as far forwards as the inferior and outer part of the lens, to which, however, it has no special attachment. Its form is quadrilateral. By its outer border it is attached to the sclerotic coat and optic nerve, following the oblique course of the latter through the tunics of the eye; this is between one. fifth and one-fourth of an inch in length. (It may be observed that in birds the optic nerve perforates the sclerotic coat on its posterior and outer side.) Its inner border is free in the substance of the vitreous humour, and not quite so long as the former. In the eyes of some animals this border is very short, and the pecten is then of a triangular figure; the distance between these two borders- that is, its breadth, is greatest at its middle, being about one-tenth of an inch; the surfaces of the pecten are so placed that one looks ontwards and a little upwards; the other, of course, in the opposite directions. After it has been cut from a band of fibrous tissue, which connects it to the sclerotic coat, and drawn out under the microscope, it will be seen in the fowl and the pigeon to consist of about twenty oval folds, plaited like the folds of a fan, each plait, at its middle, which is its broadest part, being about one-fiftieth of an inch in depth, so that as two layers of membrane form each plait, the entire organ consists of nearly oneinch in length of vascular membrane. The whole extent of this membrane supports a rich plexus of capillaries, resembling in their disposition and size tho $\mathrm{e}$ of the ciliary processes; it is covered also more or less by pigment epithelium. The vessels enter and leave it at its attached border. Now, considering the facts just stated, especially the manner in which the pecten is situated in the very substance of the vitreous humour, it will be evident that when this humour is compressed in consequence of the action of the ciliary muscle upon the choroid coat, the vessels of the pecten will be compressed also; and that, unless there be some express contrivance to prevent the escape of the blood from them, which there is no reason whatever to suppose, they will be more or less emptied of their blood, according to the degree of pressure to which they are subjected. It is further evident, considering the manner in which the pressure is exerted upon the vitreous humour, and the position of the pecten, that after the vitreous humour has been made to take up the space before occupied by the fluid contents of the vessels of the pecten, its form will be altered, being more flattened laterally, but rendered more convex posteriorly - that is, the quantity of fluid before contained in the vessels of the pecten, being subtracted from the entire mass of vitreous humour, the latter will have become pressed into a smaller spheroid, with its transverse axis shortened, whilst its antro-posterior one remains the same.

Now, it is admitted by all physiologists that the image of visible objects is in some part of the retina; therefore, the rays of light, to have arrived at this part, must have first emerged at the posterior surface of the vitreous body, and then crossed the serous space between the tunica vitria and the retina. It is certain that these rays, after their emergence, do not enter a denser refracting medium, or they would have been dispersed, and no image could have been formed; hence they may be considered to have taken their last direction at the instant of their emergence at the posterior surface of the vitreous body; and therefore, as the refracting power of lenses composed of the same material is in proportion to their degree of convexity, (Wood's Optics, 71,) so the rays emerging at the posterior surface of the vitreous body mnst be refracted most when its convexity is greatest, and vice vers $\vec{a}$; and thus the rays of light are brought to a focus soonest when the ciliary muscle is in action: and in this way the pecten is made to contribute to the same end, thongh in a different way, as the ciliary processes, and to assist these in a form of eye where perfect adjustment is inadmissible solely by the motion of the lens. From the mobility of the retina, and the manner in which it is adapted to the posterior surface of the vitreons body, it is almost certain that these will always remain parallel and equidistant, whatever may be the change in the convexity of the latter, and therefore that the law of refraction, as in. fluenced by convexity, will not be in the least affected in its application by the change of curvature which the vitreous humour will undergo from the effect of pressure upon the pecten. It has been noticed that the lens is less perfect in the bird than in the mammal; so the vitreous humour, having more to do in the former than in the latter, is more so. The vitreous humours of the fowl and sheep, dried under precisely the same circumstances, lost different quantities of fluid; the sheep lost 98 per cent., and the fowl only 93 . We may now also see a reason why there should be a stronger ciliary muscle in the bird, and other animals having eyes of a similar construction, than in the maminal; and lastly, according to this explanation of the function performed by the ciliary processes, and by the pecten, in the adjustment of the eye to distinct vision, whenever we meet with animals having a very small head, but comparatively large eyes, we may expect that they will have these organs of a flattened form, with the sclerotic coat strong anteriorly; a large ciliary muscle, probably a lens of inferior density; a very dense vitreous humour; small ciliary processes, and a pecten.

Physiologists, in treating of the adaption of the eye to distances, have attributed much to change of the convexity of the cornea, and but little or nothing to that of the posterior surface of the vitreous humour, although the effect in altering the focal distance of the eye is the same. It is true that the vitreous humour is very near to the retina, but still there is a serous space between them, as can be demonstrated by the facility with which air or fluid can be injected into it, especially at the posterior part of the eye; besides, there is, between the tunica vitria and the retina, a layer of very transparent cells. The minuteness of this space does not, however, affect the principle upon which the posterior part of the vitreous humour acts as a lens, since it is at the instant of emergence that the last direction is given to the emergent rays, and therefore the degree of refraction which these rays suffer will be in proportion to its convexity; and the position of the image with respect to the retina must vary accordingly.

\section{ON A CASE OF DEATH FROM RUPTURE OF THE UTERUS.}

Bx J. BERNCASTLE, M.D., M.R.C.S., \&c., London.

ON the 9th of September last, I was called, at eight A.M., to a woman in labour, who had engaged a midwife to attend her. On my arrival at the house I learned that the midwife had been sent for in the night; that she stayed with the patient until 
three A.M., when she said that it was no use her staying any longer, as it might be a month before the labour would come on; and she left the patient accordingly. Between three o'clock, when the midwife left, and eight, it appears that labour had come on, for I found the head of a full-grown dead child protruding, and the pains coming on regularly; with the usual assistance the child was born about a quarter of an hour after my arrival. The foetus had been dead at least a fortnight. The placenta followed quickly; there was no hæmorrhage; the uterus contracted immediately, and could be distinctly felt, like a cricket-ball, beneath the abdominal parietes. Having applied a bandage, and given an opiate, with the usual instructions that she was not to be moved, and was to be kept perfectly quiet, I left the patient remarkably well and cheerful, free from pain, talkative, and not having the appearance even of having been delivered, so well was she in all respects. Two hours afterwards I was summoned in great haste to see her again, as she was dying. I went immediately, and to my great surprise found her speechless, cold, without any pulse, deadly pale, covered with a clammy perspiration - in fact, "in articulo mortis." No hæmorrhage appeared to have taken place, unless it was internal, which was the only cause I could imagine as accounting for such untoward symptoms. Stimulus was administered, but was of no avail, and she died an hour after being thus attacked.

It appears that the midwife, not knowing that she had been confined, called at about eleven to know how she was going on, when the deceased was very angry with her for having left her in the night, and ordered her to go away; that immediately after this sudden excitement she was seized as above described, although up to that period she was exactly in the very favourable state in which I first left her. I found also that she had had, before this one, two children born dead and two miscarriages, and that she had for years been suffering from pain in the uterine region, and had been under medical treatnent for the same.

Having mentioned the case to a medical friend of great experience in uterine disease, he expressed a wish to be present at the post-mortem examination; supposing, like myself, that internal hæmorrhage would be discovered as the cause of death.

Post-mortem examination.-Body that of a well-formed, stout woman.-Chest: Heart and lungs perfectly healthy.Abdomen: Liver, kidneys, and other abdominal viscera, all healthy, but pale. The uterus was large, uncontracted, and rose above the umbilicus. On examining the appearances presented about the lower part of the uterus, a dark, extravasated appearance was observed on the right side, at the situation of the right lateral ligament. On removing the uterus, which was done by cutting throngh its neck, a great quantity of blood escaped, and a number of clots, amounting to about two pounds, were discovered. The internal surface of the nterus was now exposed; but excepting that it was not contracted in the usual manner after parturition, it did not present any abnormal appearance. There was no effusion of blood in the cavity of the uterus itself. The neck of the nterus was now particularly examined, and it was found to present a ragged and ulcerated appearance, and was covered with blood, partly coagulated and partly fluid. A great quantity of this blood lay immediately beneath the reflexion of peritonæum passing from the uterus to the bladder.

It appeared that there was an ulcerated condition of the uterus, which had probably existed for several years; and after the labour, it is probable that the wall of the neck of the uterus gave way, causing an effusion of blood, not only into the neck of the uterus, but also into the cavity of the abdomen, beneath the peritonceum; and that this sudden effusion of blood was the cause of the woman's death.

A question arises as to the period at which this rupture of the uterus occurred. Was it during labour, or during the excitement caused by the midwife's visit, when the symptoms first gave evidence of the accident? If it happened during labour, then it must be admitted that such an accident can take place and remain unnoticed by either patient or attendants for several hours: which is hardly probable, and contrary to the views of the highest medical authorities on this subject. In referring, amongst others, to Dr. Blundell's work, I find that he describes the symptoms of ruptured nterus thus:"The woman, who seemed to be doing very well previously, perhaps exclaims that something has yielded, and then the countenance falls, the stomach vomits, the extremities become cold, the pulse rises to 130 or 140 in a minute, the pains become small, weak, and irregular; in a word, death seems to have already seized upon its victim."
Nothing of this was observed for two hours after the confinement of this patient, nor until the sudden visit of the midwife produced a state of excitement which was immediately followed by a state very similar to that described by the author quoted. The question then remains-Did this rupture occur during the pains of labour, and remain unnoticed, or did it take place after the visit of the midwife, at which time only any evidence of it began to appear?

Albany-street, Regent's-park, 1951.

PRACTICAL OBSERVATIONS ON

DISORDERS AND DISEASES OF EUROPEANS ON THEIR RETURN FROM TROPICAL CLIMATES.

BY JAMES RANALD MARTIN, F.R.S.,

SURGEON, BENGAL ARMY, RETIRED; LATE PRESIDENCY-SURGEON, AND SURGEON TO THE NATIVE hOSPITAL, CALCUTTA.

(Continued from $p .55$.)

ON THE TREATMENT OF INVALIDS SUFFERING FROM THE SEQUELAR TO THE FEVERS OF TROPICAL CLIMATES.

CASE 2.-Major —-, of the Indian army, aged forty, had served twenty-two years in India. For the first sixteen years his health had been good; but during the last six years he was employed in field-surveying, the duties of which necessarily exposed him to great variations in temperature and humidity, as well as to the malarious influences. His constitution, originally delicate, appeared indeed to have been improved and invigorated during the first ten years of residence in India. After that his health remained stationary till six years ago, when he was seized, in the month of May, with the ardent remittent fever of the hot dry season. Violent determination to the head, with tumidness and pain of the right hypochondrium, took place immediately, accompanied by delirium. He was freely bled, generally and locally, and took calomel, sudorifics, and purgatives, in large doses. His recovery was rapid, and apparently complete. By the month of August he had regained his usual health, when acute bilious diarrhœa came on, rendering mild mercurials, with ipecacuanha, laxatives, and low diet, necessary. Again he recovered rapidly, and in the end of October proceeded on his duties of surveying.

The country was jungly, and the soil remained moist and marshy from the previous annual rain. His public establishment and private servants contracted intermittent fevers, but by the aid of small doses of quinine, and by selecting elevated grounds of encampment, Major — hoped to escape. He did escape until the end of January, when he was attacked with severe fever of the continued form, accompanied by jaundice. He was again bled moderately, and used calomel, antimony, purgatives, and low diet; but his recovery was slow. From this time to the present, he has never been in good health. Every year he has been more or less harassed by fevers, sometimes continued in type, at others intermittent, and his health and strength have been gradually reduced. He was urged to go to sea, ox to return to Europe, but circumstances of a private nature prevented his doing either. At length, and after years of distress and suffering, he came home in the month of February, worn out with fever. He was now greatly exhausted in all the functions of the body and mind, and in a state of great emaciation. The abdomen was hard and flattened, the abdominal muscles being in a state of extreme tension all over, and this state, owing to neuralgic pains, was rather increased by tactile examination and percussion. All seemed hard beneath, but I could not perceive that any one region was more indurated than another, and there was no tumidness in either hypochondrium. The pulse and respiration were slow and feeble, the heart flaccid, and sluggish in its movements; there was a frequent hacking, dry cough. The complexion was of a dingy parchment hue, the skin being extremely harsh and dry to the tonch; the hair was of a mossy dryness, its growth being imperceptibly slow; the nails were exfoliated, and crumbling off-all indicating that nutrition was at the lowest ebb. The intestinal secretions were scanty, pale, and fotid; the urine of a natural appearance, occasionally linfpid. For years the bowels had not acted without the aid of medicine. He had no appetite, and experienced great oppression, with sense of distention, after the smallest meal. The sleep was disturbed and unrefreshing; the general habit was anæmic, and the mind greatly depressed.

To promote the functions of depuration, and to impart tone to the digestive organs, Major — was directed to use the nitromuriatic acid bath three times a week, just before going to bed, while a combination of the nitro-muriatic acid with tinc- 